\title{
Childhood Onset Systemic Lupus Erythematosus with Direct Coombs Test Positive in the Absence of Hemolytic Anemia - A Rare Case Report
}

\author{
Vani Sreekumar* and Mangaiyarkarasi \\ Blood Bank, Coimbatore Medical College, Coimbatore
}

\begin{abstract}
Systemic Lupus Erythematosus being a chronic autoimmune multisystem inflammatory disease, affects predominantly women of reproductive age group. Childhood - Onset Systemic Lupus Erythematosus is a rare disease with an incidence of 10\% - 20\%. Positive direct Coombs test in the absence of hemolytic anemia indicates high disease activity and severe renal damage. Herein we report a case of 11-year-old female child diagnosed as Systemic Lupus Erythematosus with positive direct Coombs test in absence of hemolytic anemia which is very rare.
\end{abstract}

Keywords: Systemic Lupus Erythematosus, Direct Coombs Test, Hemolytic Anemia

\section{Introduction}

Systemic Lupus Erythematosus is a clinically heterogenous disease which is autoimmune in origin and a multisystem disease, characterized by the presence of autoantibodies directed against nuclear antigens ${ }^{[1]}$. SLE is more common in women than men and has a predilection for women in their child bearing years. Childhood Systemic Lupus Erythematosus is rare with incidence of $0.3-0.9$ per 100000 children per year, and a prevalence of $3.3-24$ per 100000 children ${ }^{[2]}$ The clinical course of childhood Systemic Lupus Erythematosus is severe and the prevalence of lupus nephritis, hematologic anomalies, photosensitivity, neuropsychiatric and mucocutaneous involvement are high.

Direct Coombs test positivity in Systemic Lupus Erythematosus can be either associated with Hemolytic anemia or in the absence of Hemolytic anemia. A positive Coombs test in Systemic Lupus Erythematosus patients without hemolytic anemia shows higher SLE disease activity index (SLEDAI), increased immune complex levels and anti - DNA titers, with lower renal response rate. ${ }^{[3]}$ Therefore, positive Coombs's test in the absence of hemolytic anemia may be useful as a biomarker for assessing disease activity and therapeutic response.

\section{Case Report}

A 11-year-old girl was admitted to our hospital with complaints of low-grade fever, swelling of limbs and face of one week duration. She also had oral ulcer and reddish spots over lower limbs for two days. She had history of surgery operated for left side cervical swelling four years ago, the details of which are not available. There is no positive family history and she had a normal developmental history. A written informed consent was obtained.

On examination she was conscious and oriented. She had pallor of skin and conjunctiva. She had facial puffiness, swelling of hands, pedal edema and multiple petechiae over legs. She also had gum bleed and oral ulcers. Her weight was $23 \mathrm{~kg}$ and height was $132 \mathrm{~cm}$. Vital signs were: heart rate $132 / \mathrm{min}$, respiratory rate $36 / \mathrm{min}$, blood pressure 100/60mmHg, spo2 $100 \%$. Palpable lymph nodes were detected in cervical region and inguinal region. On ophthalmology examination patient had periorbital edema and multiple petechial hemorrhages in the posterior pole of retina.

\section{Investigations}

Blood test revealed severe anemia with hemoglobin 6gm/ $\mathrm{dl}$, leucopenia and thrombocytopenia with platelet count $5000 /$ microliter. White blood count is $3500 /$ microliter. Peripheral blood film revealed dimorphic anemia with thrombocytopenia. There was no evidence of hemolytic anemia. ESR was elevated - $121 \mathrm{~mm} / \mathrm{hr}$. Lactate dehydrogenase levels were within normal limits. Renal function test revealed increase in urea $60 \mathrm{mg} / \mathrm{dl}$. Serum creatinine was $1.0 \mathrm{mg} / \mathrm{dl}$, sodium $149 \mathrm{mEq} / \mathrm{L}$ and potassium $4.1 \mathrm{mmol} / \mathrm{L}$. Urine examination showed trace amounts of protein, 6-8 pus cells/hpf, 3-5red cells/hpf and 4-6 epithelial cells/hpf. Liver function tests were within normal limits. Chest x-ray showed cardiomegaly. Ultrasound abdomen showed hepatosplenomegaly with moderate ascites and bilateral mild increases in renal echoes. 
Direct Coombs test was positive. Immunological tests were conducted and the results showed Anti - nuclear antibody (ANA) with value 2.07 OD ratio which is considered positive. The anti - double stranded DNA antibody test which is regarded as specific marker for systemic lupus erythematosus was done and yielded a positive result of 4.26 OD ratio. Renal biopsy was not done as the patient was not willing for the procedure.

This case was confirmed based on the laboratory findings and the clinical presentation as Childhood onset systemic lupus erythematosus with direct Coombs test positive in the absence of hemolytic anemia and with renal involvement. The patient was treated with hydroxychloroquine $200 \mathrm{mg}$ half $\mathrm{OD}$ and prednisolone $1 \mathrm{mg} / \mathrm{kg} /$ day. The improvement was first detected in the laboratory findings followed by clinical improvement and then the patient was discharged. Follow up of the patient could not be done.

\section{Discussion}

Systemic lupus erythematosus is a chronic autoimmune disease characterized by antibodies which are against self - antigens causing inflammation leading to tissue damage ${ }^{[4]}$. Systemic lupus erythematosus affects multiple organ systems. It is more common among women and African descendants ${ }^{[5]}$.

One of the most common systemic autoimmune disease in children is Systemic lupus erythematosus. ${ }^{[6]}$ Pediatric systemic lupus erythematosus is a rare condition, representing approximately $10 \%$ of SLE cases. Female children are predominantly affected with peak age of onset being 12 years. ${ }^{[7]}$ Pediatric SLE shows higher frequency of renal, hematological and neuropsychiatric involvement than adult SLE.

Direct Coombs test positivity in systemic lupus erythematosus is seen either associated with autoimmune hemolytic anemia or in the absence of hemolytic anemia. ${ }^{[8]}$ Both anti - erythrocyte antibody which is bound to the surface of erythrocytes and immune complex binding to CR1 on erythrocytes are detected by Direct Coombs test. ${ }^{[9]}$ The hallmark of Autoimmune hemolytic anemia is binding of anti - erythrocyte antibody to erythrocytes whereas immune complex binding to CR1 is not associated with hemolysis. Overexpression of immune complex is one of the main pathogenic mechanism in SLE. Since erythrocytes clear immune complex via CR1, the direct Coombs test can be positive without hemolysis in patients with overexpression of immune complex ${ }^{[3]}$.

In the Systemic Lupus Erythematosus International Collaborating Clinics/American College of Rheumatology 2012, the direct Coombs test in the absence of hemolytic anemia has been newly included as a criterion for systemic lupus erythematosus. ${ }^{[10]}$ Therefore excessive immune complex production may be indicated by positive direct Coombs test in the absence of hemolytic anemia.

A study done by $\mathrm{H}$. Hanaoke et al. at St. Marianna University school of Medicine, Kawasaki, Japan showed that $5.8 \%$ of systemic lupus erythematosus patients had a positive direct Coombs test in the absence of hemolytic anemia and four patients had renal involvement. Our case is similar to above study with positive direct Coombs test in the absence of hemolytic anemia and renal involvement. [3]

The individuals with positive direct Coombs test in the absence of hemolytic anemia have shorter disease duration, high circulating immune complexes, higher anti - DNA antibody titers, lower renal response rate and high SLEDAI scores. ${ }^{[3]}$

In conclusion, the presence of positive Coombs test in the absence of hemolytic anemia in systemic lupus patients may indicate excessive immune complex production and thus it may be used as a biomarker for evaluating the disease activity and therapeutic response.

\section{Conclusion}

Systemic Lupus Erythematosus is a chronic autoimmune disease. Childhood onset SLE is rare. Direct Coombs test positivity in the absence of hemolytic anemia in SLE indicates high disease activity and severe renal damage. Hence positive Coombs test can be used as a biomarker for evaluating the disease activity.

\section{Acknowledgements Funding}

NIL

\section{Competing Interests}

NIL

\section{Reference}

1. Manson, J.J., Rahman, A. Systemic lupus erythematosus. Orphanet J Rare Dis 2006 Mar 27;1: 6.

2. Harry O, Yasin S, Brunner H. Childhood-Onset Systemic Lupus Erythematosus: A Review and Update. J Pediatr. May; 196: 22-30. e2.

3. Hanaoka H, Iida H, Kiyokawa T, Takakuwa Y, Kawahata K. A positive direct Coombs' test in the absence of hemolytic anemia predicts high disease activity and poor renal response in systemic lupus erythematosus. Lupus. 2018 Dec; 27(14) :2274-78.

4. Master Sankar Raj V. An unusual presentation of lupus in a pediatric patient. August 2013.Case reports in Paediatrics 2013(1): 180208; 
5. Ferraria N, Rocha S, Fernandes VS, Correia T, Gonçalves E. Juvenile systemic lupus erythematosus with primary neuropsychiatric presentation. BMJ Case Rep. 2013Jan 25.

6. Amit Kumar, Pradeep Kumar, Masuma P. Bhengra, Prabhat Kumar, Syam Sundar Chaudhary, Childhood-onset systemic lupus erythematosus - a case report, J. Pak. Assoc. Dermatol. 2016;26(1): 76- 79

7. Tucker LB, Menon S, Schaller JG, Isenberg DA. Adultand childhood-onset systemic lupus erythematosus: a comparison of onset, clinical features, serology, and outcome. Br J Rheumatol. 1995: Sep; 34(9): 866-72.
8. Mongan ES, Leddy JP, Atwater EC, Barnett EV (1967) Direct antiglobulin (Coombs) reactions in patients with connective tissue diseases. Arthritis Rheum 1967 Dec; 10(6): 502- 8

9. Inada Y, Kamiyama M, Kanemitsu T, Clark WS, Asai Y. Relationships between $\mathrm{C} 3 \mathrm{~b}$ receptor (CR1) activity of erythrocytes and positive Coombs' tests. Ann Rheum Dis. 1986 May;45(5):367-72.

10. Petri M, Orbai AM, Alarcón GS, et al. Derivation and validation of the Systemic Lupus International Collaborating Clinics classification criteria for systemic lupus erythematosus. Arthritis Rheum. 2012; Aug 64(8): 2677-86.

*Corresponding author:

Vani Sreekumar, D1, Orchid Covent Apartments, Parameshwaran Pillai Layout, Pappanaickenpalayam, Coimbatore - 641037

Phone: +91 9487556813

Email: sruthivani@gmail.com

Date of Submission $\quad: 24 / 02 / 2021$

Date of Final Revision : 17/07/2021

Date of Acceptance $\quad: 18 / 07 / 2021$

Financial or other Competing Interests: None.

Date of Publication $\quad: 30 / 07 / 2021$ 